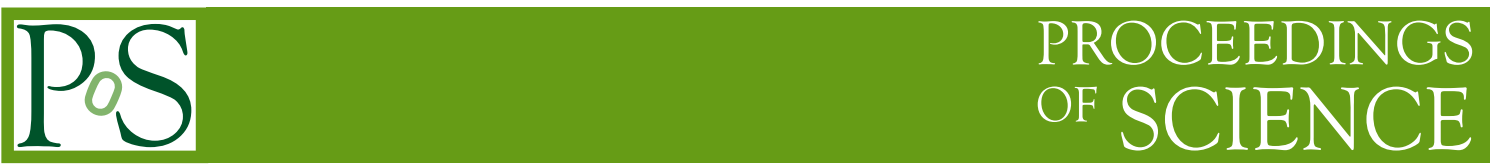

\title{
Charm-bottom baryon spectroscopy
}

\author{
Zachary Brown ${ }^{* a b}$ William Detmold, ${ }^{a b}$ Stefan Meinel, ${ }^{a}$ and Kostas Orginos ${ }^{a b}$ \\ ${ }^{a}$ Department of Physics, College of William \& Mary \\ Williamsburg, VA 23187-8795 \\ ${ }^{b}$ Thomas Jefferson National Accelerator Facility \\ Newport News, VA 23606 \\ E-mail: zsbrown@email.wm.edu, wdetmold@wm.edu, smeinel@wm.edu, \\ kostas@wm. edu
}

\begin{abstract}
The arena of doubly and triply heavy baryons remains experimentally unexplored to a large extent. This has led to a great deal of theoretical effort being put forth in the calculation of mass spectra in this sector. Although the detection of such heavy particle states may lie beyond the reach of experiments for some time, it is interesting to compare results between lattice QCD computations and continuum theoretical models. Several recent lattice QCD calculations exist for both doubly and triply charmed as well as doubly and triply bottom baryons. In this work we present preliminary results from the first lattice calculation of the mass spectrum of doubly and triply heavy baryons including both charm and bottom quarks. The wide range of quark masses in these systems require that the various flavors of quarks be treated with different lattice actions. We use domain wall fermions for $2+1$ flavors (up down and strange) of sea and valence quarks, a relativistic heavy quark action for the charm quarks, and non-relativistic QCD for the heavier bottom quarks. The calculation of the ground state spectrum is presented and compared to recent models.
\end{abstract}

Sixth International Conference on Quarks and Nuclear Physics

April 16-20, 2012

Ecole Polytechnique, Palaiseau, Paris

${ }^{*}$ Speaker. 


\section{Motivation}

Experimental observations of charmed and bottom baryons date back to the mid 1970's with the discovery of the $\Lambda_{c}^{+}$, and to the early 1990's with the discovery of the $\Lambda_{b}$. Since that time, the experimental spectrum has filled out with many very precise measurements of the ground state energies of singly charmed and bottom baryons. In the past 10 years, results from BaBar and Belle, as well as the D0 and CDF experiments at Fermilab have provided more precise measurements of heavy flavour ground and excited states as well as a first glimpse of several new states, most notably the controversial $\Omega_{b}$ at CDF [1] and D0 [2] and the $\Xi_{c c}$ at SELEX [3, 4]. Although the former controversy has been resolved by a more recent measurement at the LHCb [5], the latter observation has still received no experimental verification to date, and is generally in disagreement with most current theoretical results by $\sim 200 \mathrm{MeV}$. It is controversies like these, as well as the prospect of observations of new states that make this an exciting time for lattice QCD. The opportunities for heavy flavour spectroscopy from the lattice are twofold: 1) Lattice results can help to resolve controversies like the ones presented above (and provide an excellent comparison with current experimental measurements) and 2) Lattice results can help to provide predictions for as yet unobserved states. The predictive power of lattice QCD is especially exciting in the context of the upcoming increase in operating energy at the LHC scheduled for 2014. It is with this motivation that we present the first lattice calculation of the charmed bottom baryon spectrum.

\section{Heavy Quarks in Lattice QCD}

Lattice QCD is a discretized version of the Feynman path integral formulation of QCD. By defining QCD on a four dimensional Euclidean space time lattice with lattice spacing $a$, we regulate the continuum theory with an ultraviolet cutoff at energies greater than $\frac{1}{a}$. By necessarily performing calculations in a finite volume (with spatial length $L$ and periodic boundary conditions), the lattice also introduces an infrared cutoff at energies less than $\frac{1}{L}$. To make contact with QCD, extrapolations in both the lattice spacing and lattice volume are necessary. The desired scale hierarchy for lattice calculations is:

$$
a \ll\left\{\frac{1}{m_{h}}, \frac{1}{p_{\text {hadron }}}, \frac{1}{\Lambda_{Q C D}}, \frac{1}{m_{\pi}}\right\} \ll L
$$

where $m_{h}$ is the mass of a heavy quark. Due to the increase in computational cost associated with reducing the lattice spacing and pion mass, this hierarchy is currently not computationally feasible, and it is common to work with the following hierarchy instead:

$$
\frac{1}{m_{h}}<a \ll\left\{\frac{1}{p_{\text {hadron }}}, \frac{1}{\Lambda_{Q C D}}, \frac{1}{m_{\pi}}\right\}<L,
$$

with $m_{\pi}>m_{\pi}^{p h y s}$. In order to account for the unphysical pion mass, chiral perturbation theory is often used to perform extrapolations to the physical point. Heavy quarks are treated differently due to computational limitations. With standard fermion actions, the required lattice spacings to correctly resolve charmed and bottom quarks are $a<0.06 \mathrm{fm}$ and $a<0.02 \mathrm{fm}$ respectively, while current typical lattice spacings lie in the range of $0.08-0.12 \mathrm{fm}$. Because of this, heavy quarks are 
often handled through the use of effective theories for heavy quarks and specialized discretizations of the QCD action. Two such treatments of heavy quarks on the lattice (used in the current work) are non-relativistic QCD (NRQCD) [6], and the relativistic heavy quark action (RHQ) [7]

NRQCD is an effective field theory in which the hard modes at or above $m_{h}$ have been integrated out. The action contains local operators of increasing dimension, which are suppressed by increasing powers of the typical heavy-quark velocity. Lattice NRQCD is a discrete version of continuum NRQCD, and requires a cutoff $a^{-1}<m_{h}$. This requirement, together with the rate of convergence of the heavy-quark expansion, means that lattice NRQCD is well-suited for bottom quarks, but not for charm quarks. For the charm quarks, we use the RHQ action, which is a Wilson-like action with improvement terms (proportional to powers of the lattice spacing, $a$ ) and an anisotropy $v$ between the spatial and temporal directions. By tuning this anisotropy, the heavy quark dispersion relationship, which is badly broken due to the scale discrepancy between the heavy quark mass and the lattice spacing, can be restored.

\section{Lattice Spectroscopy}

Masses are extracted from lattice calculations by looking at the Euclidean time behaviour of two point correlations functions of interpolating operators $O(\vec{x}, t)$ that have the quantum numbers of the state of interest. Explicitly, we have the relation:

$$
C(t)=\sum_{\vec{x}}\langle O(\vec{x}, t) \bar{O}(0,0)\rangle=\sum_{i} A_{i} e^{-m_{i} t},
$$

where the $m_{i}$ are the masses of all states that couple to the chosen operators. At large times, the ground state mass dominates the correlator signal and a single exponential fit is often sufficient to extract the ground state energy at large times provided there are no other sources of contamination to the signal in this region.

For the current work, charmed bottom interpolating operators are of the form

$$
O_{5}=\varepsilon_{a b c}\left(C \gamma_{5}\right)_{\beta \gamma} q_{\beta}^{a} q_{\gamma}^{\prime b} q_{\alpha}^{\prime \prime c}, \quad O_{j}=\varepsilon_{a b c}\left(C \gamma_{j}\right)_{\beta \gamma} q_{\beta}^{a} q_{\gamma}^{\prime b} q_{\alpha}^{\prime \prime c},
$$

where $q\left(q^{\prime}, q^{\prime \prime}\right)$ are the quark flavours u,d,s,c,b, and Greek and Roman indices used for spin and color degrees of freedom respectively. The operators $O_{j}$ can be projected to either $J=1 / 2$ or $J=3 / 2$.

\section{Lattice Details}

The spectroscopy calculation was performed using field configurations generated by the RBC UKQCD Collaboration [8,9]. These configurations use the Iwasaki gauge action and include 2+1 flavours of light domain wall fermions (with $L_{s}=16$ ), with $a^{-1} \sim 1.76 \mathrm{GeV}, 2.33 \mathrm{GeV}$ for coarse and fine lattice spacings. The range of pion masses for these ensembles is $295 \mathrm{MeV}-352 \mathrm{MeV}$, and we compute propagators corresponding to valence pion masses in the range $227 \mathrm{MeV}-352$ $\mathrm{MeV}$. The strange quark mass for the ensembles is approximately $10 \%$ above the physical point, however we also generate strange-quark propagators with slightly lower mass to interpolate to the physical value. 
The RHQ action is used for the charm quarks, with tree level tadpole improved values for the coefficients $c_{E}$ and $c_{B}$. This leaves two free parameters which we nonperturbatively tuned: the RHQ anisotropy " $v$ " and the bare quark mass " $m_{0}$ ". These two parameters are tuned simultaneously to restore the $J / \Psi$ dispersion relation:

$$
c^{2}=\frac{E_{|p|=1}^{2}-E_{|p|=0}^{2}}{p_{|p|=1}^{2}}=1,
$$

as well as match to the experimental value of the spin averaged charmonium mass: $\bar{M}=\frac{1}{4} M_{\eta_{c}}+$ $\frac{3}{4} M_{J / \Psi}$. It should be noted here that we choose to use Eq. (4.1) in tuning the dispersion relation over performing a linear fit for a larger number of momenta.

The lattice NRQCD action used here for the bottom quarks is accurate through order $v^{4}$, $\Lambda_{\mathrm{QCD}}^{2} / m_{b}^{2}$ [6], and is tadpole improved using the Landau gauge mean link. The matching coefficients $c_{i}$ are set to their tree-level values, except for $c_{4}$ (which controls hyperfine splittings). For $c_{4}$, the one-loop results are used [10], kindly provided by Tom Hammant for the case of the Iwasaki gauge action used here. The bottom quark mass is tuned nonperturbatively to reproduce the correct spin-averaged kinetic mass for the bottomonium $1 S$ states.

\section{Results}

In Figure 1 we present the low lying spectrum of baryons containing charmed and bottom quarks. The masses shown are calculated from mass splittings with respect to experimental masses from the Particle Data Group. For states containing light quarks, $n_{c}$ charm quarks and $n_{b}$ bottom quarks we have:

$$
M_{O}=M_{O}^{\text {latt }}-n_{c} M_{\Lambda_{c}}^{\text {latt }}-n_{b} M_{\Lambda_{b}}^{\text {latt }}+n_{c} M_{\Lambda_{c}}^{P D G}+n_{b} M_{\Lambda_{b}}^{P D G}
$$

This particular choice is made in an attempt to cancel some of the quark mass dependence, as well as the NRQCD energy shift, from the values we present. For states containing only heavy quarks, $M_{\Lambda_{b}}\left(M_{\Lambda_{c}}\right)$ is replaced by $\frac{1}{2} M_{\Upsilon}\left(\frac{1}{2} M_{J / \Psi}\right)$. The preliminary results presented here are at a finite lattice spacing of $\sim 0.11 \mathrm{fm}$, and a valence pion mass of $\sim 270 \mathrm{MeV}$. As stated in the previous section, the calculation was performed at two lattice spacings with several different pion masses which allows for extrapolations to both the continuum $(a \rightarrow 0)$ and chiral limits.

In light of the fact that no previous lattice calculations of the mixed charmed bottom baryon exist, it is interesting to compare this lattice calculation with other theoretical results that exist from calculations with various models. In Figure 2 we compare our value of $\Omega_{c b}^{(*)}$ to several other theoretical values that exist in the literature. Because the $\Omega_{c b}^{(*)}$ lacks any light valence quarks, the pion mass dependence is expected to be very weak (entering only at the level of loop diagrams). In comparing the various models to our lattice calculation we find agreement with only the results from Ref. [14]. This could be indicative of the shortcomings of calculations using various flavours of the quark model, although there is still room for movement in our lattice values from interpolating to the physical strange quark mass. 


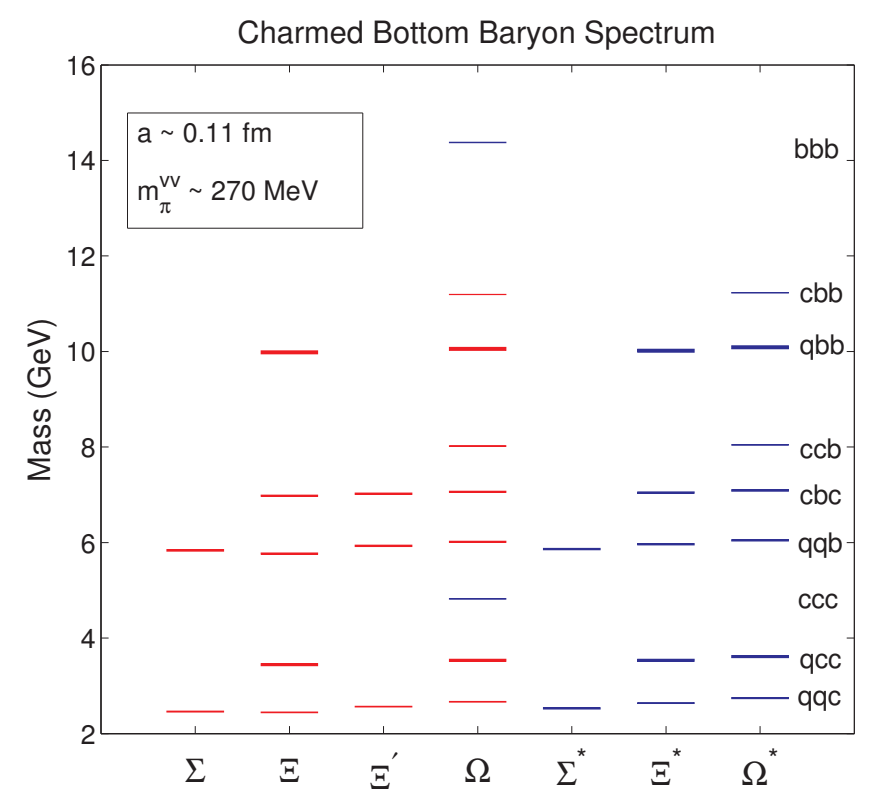

Figure 1: Charmed bottom spectrum at non-zero lattice spacing and pion mass of $m_{\pi}^{v v} \sim 270 \mathrm{MeV}$. Blue lines are $J=1 / 2$ and red are $J=3 / 2$.
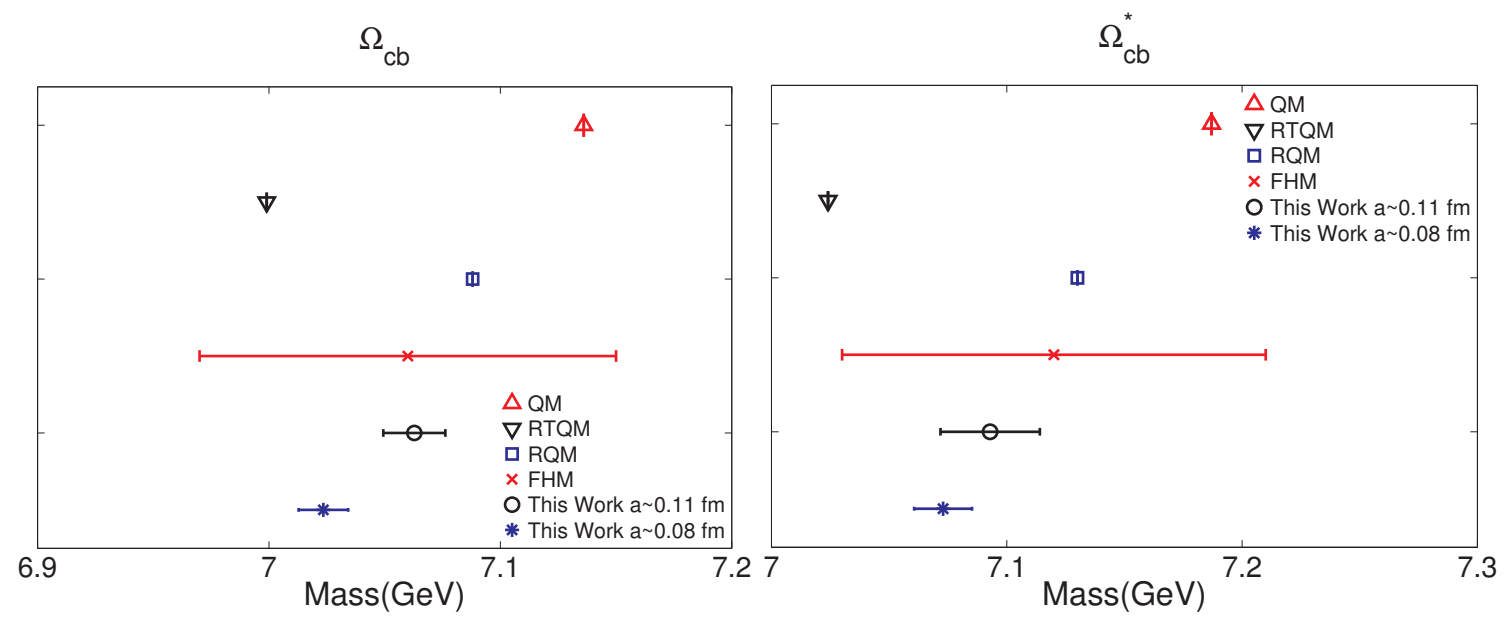

Figure 2: Comparison of $\Omega_{c b}^{(*)}$ to various theoretical models. The uncertainty quoted for the lattice results are statistical only; systematic uncertainty from fitting is expected to contribute less than the statistical error. The other calculations are as follows: QM is a quark model calculation presented in [11], RTQM is a relativistic three quark model result from [12], RQM is relativistic quark model from [13], and FHM is from [14] using the Feynman-Hellmann theorem.

\section{Conclusions}

We have presented preliminary results from the first lattice calculation of the low lying charmed bottom baryon spectrum, motivated by recent observations of new charm and bottom baryons and 
their resonances. We present a comparison of several states to other theoretical models and find relatively weak agreement with all but one previous calculation. Future plans include performing extrapolations to the continuum and chiral limits and an extrapolation to the physical strange quark mass to make the connection with QCD.

\section{Acknowledgments}

William Detmold and Kostas Orginos were supported in part by DOE grants DE-AC05-06OR23177 (JSA) and DE-FG02-04ER41302. William Detmold and Stefan Meinel were also supported by DOE OJI grant DE-SC0001784 and Jeffress Foundation Research grant J-968. Zachary Brown was supported by DE-FG02-04ER41302 and the JSA Jefferson Lab Graduate Fellowship Program.

\section{References}

[1] Aaltonen, T. and others., Observation of the Omega(b)-Baryon and Measurement of the Properties of the $\Xi_{b}^{-}$and $\Omega_{b}^{-}$Baryons, Phys.Rev. D80 (072003) 2009 [hep-ex/ 0905 . 3123]

[2] V. M. Abazov et al., Observation of the Doubly Strange b Baryon $\Omega_{b}^{-}$, Phys. Rev. Lett. 101 (232002) 2008

[3] M. Mattson et al., First observation of the doubly charmed baryon $\Xi_{c c}^{+}$, Phys. Rev. Lett. 89 (112001) 2002

[4] A. Ocherashvili et al., Confirmation of the double charm baryon $\Xi_{c c}(3520)^{+}$via its decay to $p$ D+ K-, Phys. Lett. B628 (18-24) 2005

[5] D. Milanes [LHCb Collaboration], Heavy flavor production and spectroscopy at LHCb, arXiv: 1201.4717.

[6] G. P. Lepage et al., Improved nonrelativistic QCD for heavy quark physics, Phys. Rev. D 46, 4052 (1992) [arXiv:hep-lat/9205007].

[7] Christ, Norman H. and Li, Min and Lin, Huey-Wen, Relativistic Heavy Quark Effective Action, Phys.Rev. D76 (074505) 2007 [hep-lat/ 0608006 ]

[8] Allton, C. and others, 2+1 flavor domain wall QCD on a $(2 \mathrm{fm})^{3}$ lattice: Light meson spectroscopy with $L(s)=16$, Phys.Rev. D76 (014504) 2007 [hep-lat/ 0701013 ]

[9] Aoki, Y., et al., Continuum limit physics from $2+1$ flavor domain wall QCD, Phys. Rev. D83 (074508) 2011

[10] T. C. Hammant, A. G. Hart, G. M. von Hippel, R. R. Horgan, and C. J. Monahan, Radiative improvement of the lattice NRQCD action using the background field method and application to the hyperfine splitting of quarkonium states, Phys. Rev. Lett. 107, 112002 (2011) [arXiv: 1105.5309 ].

[11] Roberts, W. and Pervin, Muslema, Heavy baryons in a quark model, Int.J.Mod.Phys. A23 (2817-2860) 2008 [nucl-th/0711.2492]

[12] Martynenko, A.P., Ground-state triply and doubly heavy baryons in a relativistic three-quark model, Phys.Lett. B663 (317-321) 2008 [hep-ph / 0708 . 2033]

[13] Ebert, D. and Faustov, R.N. and Galkin, V.O. and Martynenko, A.P., Mass spectra of doubly heavy baryons in the relativistic quark model, Phys.Rev. D66 (014008) 2002 [hep-ph / 0201217 ]

[14] Roncaglia, R. and Lichtenberg, D.B. and Predazzi, E., Predicting the masses of baryons containing one or two heavy quarks, Phys.Rev. D52 (1722-1725) 1995 [hep-ph/9502251] 